\title{
TB and MDR/XDR-TB in European Union and European Economic Area countries:
} managed or mismanaged?

\author{
G.B. Migliori****, G. Sotgiü\#,***, L. D’Ambrosio*, R. Centis*, C. Lange", \\ G. Bothamley ${ }^{+}$, D.M. Cirillo ${ }^{\S}$, S. De Lorenzo ${ }^{f}$, G. Guenther ${ }^{\uparrow}$, K. Kliiman**, \\ R. Muetterlein ${ }^{\# \#}$, V. Spinu" ${ }^{\text {"थ }}$, M. Villar ${ }^{++}$, J.P. Zellweger ${ }^{\S \S}$, A. Sandgren ${ }^{f f}$, \\ E. Huitric ${ }^{f f}$ and D. Manissero ${ }^{f f}$
}

ABSTRACT: In spite of the growing awareness of emerging drug-resistant Mycobacterium tuberculosis, the extent of inappropriate tuberculosis (TB) case management may be underestimated, even in Europe. We evaluated TB case management in the European Union/ European Economic Area countries, with special focus on multidrug-resistant (MDR) and extensively drug-resistant (XDR)-TB, using a purposely developed, standardised survey tool.

National reference centres in five countries representing different geographical, socioeconomic and epidemiological patterns of TB in Europe were surveyed. 40 consecutive, original clinical TB case records (30 MDR/XDR-TB cases) were reviewed in each of the five countries. The findings were recorded and, through the survey tool, compared with previously agreed and identified international standards.

Deviations from international standards of TB care were observed in the following areas: surveillance (no information available on patient outcomes); infection control (lack of respiratory isolation rooms/procedures and negative-pressure ventilation rooms); clinical management of TB, MDR-TB and HIV co-infection (inadequate bacteriological diagnosis, regimen selection and treatment duration); laboratory support; and diagnostic/treatment algorithms.

Gaps between present international standards of care and the management of MDR/XDR-TB patients were identified. Training, increased awareness, promotion of standards and allocation of appropriate resources are necessary to ensure appropriate care and management as well as to prevent further emergence of drug resistance.

KEYWORDS: European Centre for Disease Prevention and Control, extensively drug-resistant tuberculosis, management, multidrug-resistant tuberculosis, tuberculosis, Tuberculosis Network European Trials Group

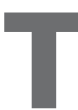

he emergence of multidrug-resistant (MDR) tuberculosis (TB), defined as in vitro resistance to isoniazid and rifampicin [1-7], and extensively drug-resistant (XDR)-TB, defined as in vitro drug-resistance to isoniazid and rifampicin as well as any fluoroquinolone and at least one of the injectable drugs (amikacin, capreomycin or kanamycin), represent a major threat to TB control at the global level [1-7].

In 2008, the World Health Organization (WHO) estimated a global prevalence of 440,000 MDR-TB cases $(390,000-510,000)$ with 150,000 deaths and an estimated 50,000 XDR-TB cases [1, 2]. 15 out of the
27 high MDR-TB burden countries are located in the European region, of which five are in the European Union (EU) and European Economic Area (EEA) [1-3].

As for MDR-TB, the emergence of XDR-TB is a manmade product, resulting, in essence, from clinical mismanagement of TB cases with levels of anti-TB drug resistance [1, 2]. There is evidence that suboptimal TB case management in parts of Europe contributes to the development of resistance to the XDR-TB-defining drugs [6-10].

In spite of the growing awareness of $\mathrm{TB}$ drug resistance, the essential variables necessary to fully
AFFILIATIONS

*WHO Collaborating Centre for Tuberculosis and Lung Diseases, Fondazione S. Maugeri, Care and Reasearch Institute, Tradate, ${ }^{*}$ Epidemiology and Medical Statistics Unit, Dept of Biomedical Sciences, University of Sassari Medical School, Sassari,

${ }^{\S}$ Emerging Bacterial Pathogens Unit, San Raffaele Scientific Institute, Milan, ${ }^{f}$ MDR-TB Dept, E. Morelli Hospital, Sondalo, Italy.

"Division of Clinical Infectious Diseases, Research Center Borstel,

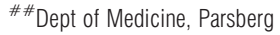
Hospital, Parsberg, Germany. +Homerton University Hospital, London, UK.

**Dept of Pulmonary Medicine, University of Tartu, Tartu, Estonia.

"MDR-TB Centre, Marius Nasta Institute of Pulmonology, Bucharest, Romania. "General Directorate of Health, Lisbon, and Lung Diseases Centre of Venda Nova, Amadora, Portugal.

${ }^{\S \S}$ Swiss Lung Association, Berne, Switzerland.

${ }^{f f}$ European Centre for Disease Prevention and Control, Stockholm, Sweden.

***These authors contributed equally.

CORRESPONDENCE

E. Huitric

Office of the Chief Scientist, European Centre for Disease Prevention and Control (ECDC), Tomtebodavägen 114 17183 Stockholm, Sweden

E-mail: emma.huitric@ecdc.europa.eu

Received:

Oct 032011

Accepted after revision:

Dec 232011 
understand MDR-TB and XDR-TB are currently not systematically collected, analysed and reported in published studies $[4,10]$. Although the international community is providing a rapid response to $\mathrm{XDR}-\mathrm{TB}$ (formulation of an emergency plan [11]; development of an instrument to support implementation and scale-up of national strategies and to assess programmatic needs $[12,13])$, no international study is available to investigate the key factor responsible for the emergence of MDR/XDR-TB, i.e. the extent of inappropriate TB case management.

The aim of the present study was to apply an ad hoc-developed, standardised comprehensive survey tool to evaluate TB case management, with special focus on MDR/XDR-TB, in the EU/ EEA [14].

\section{METHODS \\ Development of a standard tool to perform the clinical survey}

A standard tool was developed to perform the clinical survey, for which the process has previously been described [14]. In summary, the tool includes items covering the key areas of TB prevention, diagnosis and treatment. These items and questions were identified by a panel of experts and considered adequate to evaluate TB management in the selected reference centres. Specifically, a Delphi process was used to identify the elements belonging to the areas mentioned above, as well as to assign priorities to the questions proposed and to define a weighted score to the answers obtained. The key items for the questions identified by the expert panel are summarised in table 1 .

The tool is composed of three parts (Annex I) and one annex (Annex II): Part 1 summarises the features of each setting surveyed (62 items); Part 2 collects information on the individual clinical records surveyed (one line per clinical case; 141 items); Part 3 allows comparison of the key case management decisions taken on each individual case with that of internationally agreed standards (25 scored questions; standards in Annex II). The tool is included as Annex I and II (online supplementary material).

\section{Standards development}

International reference standards used to define the "correct answer", as shown in Annex II (online supplementary material), were derived from the following guidelines: the National Collaborating Centre for Chronic Conditions/British Thoracic Society guidelines [15]; the International Standards for Tuberculosis Care [16, 17]; the WHO Treatment of Tuberculosis guidelines [18, 19]; the recently developed US Agency for International Development (USAID)/Program for Appropriate Technology in Health (PATH) standardised MDR/XDR-TB assessment and planning tool $[12,20]$; the WHO MDR-TB management guidelines [21]; and the WHO policy on infection control [22]. In addition, treatment outcome definitions were used according to the criteria proposed by LASERSON et al. [23]. The criteria commonly used to define a modern approach to TB control and elimination in Europe were also used [24].

The tool was applied to each individual, original patient record examined, generating an adherence/nonadherence reply on the electronic data collection sheet and, finally, a score.

The reviewing teams, consisting of the Tuberculosis Network European Trials Group (TBNET) and local experts, were trained on how to use the tool, how to define adherence/nonadherence to the established international standards and how to assign the scores. The teams included at least one national and one international public health expert.

\section{Selection of TB reference centres}

Five MDR-TB reference institutes, identified in collaboration with national authorities (ministries of health), were identified in five countries representing four different TB epidemiological settings in the EU/EEA. As per European Centre for Disease Control and Prevention (ECDC) policy, the selected countries cannot be disclosed. The selected settings were as follows: one country each from Group 1 (former Soviet Union; intermediate TB incidence, high MDR-TB prevalence), Group 2a (northern Europe; low TB incidence, low MDR-TB prevalence), Group 2b (southern Europe; low TB incidence, low MDR-TB prevalence), Group 3 (southern Europe; intermediate TB incidence, low MDR-TB prevalence) and Group 4 (central Europe; intermediate TB incidence, low MDR-TB prevalence).

40 consecutive, original clinical TB case records were reviewed at each study site, of which 30 were MDR/XDR-TB and 10 were "other than MDR-TB" cases (i.e. the strain being susceptible, monoresistant or polyresistant). Prior to the visits, each study site retrieved the consecutive clinical files of the cases to survey following a time criterion (cases treated since January 1, 2005). All five study sites were visited between November 2009 and March 2010.

The design of the survey was approved by the scientific committee of the ECDC. No ethical approval was necessary to carry out this public health survey.

\section{Data analysis}

Data were analysed using Stata 9.0 (StataCorp, College Station, TX, USA).

TABLE 1 Key variables identified by the expert panel for the survey questionnaire

$\begin{array}{ll}\text { TB prevention } & \text { BCG vaccination; administrative measures for infection control; infection control plan; training of staff; health education of patients and contacts; } \\ \text { respiratory protection; isolation procedures; natural or mechanical ventilation } & \text { Pulmonary and extra-pulmonary TB; radiological findings; microbiological examinations including sputum smear, culture, DST for first- and } \\ \text { second-line drugs (including rapid drug resistance testing, line probe assays, nucleic acid amplification techniques); immunological findings, } \\ \text { focusing on IGRAs and TST; HIV test offered; HIV status }\end{array}$

TB: tuberculosis; BCG: bacille Calmette-Guérin; DST: drug susceptibility testing; IGRA: interferon- $\gamma$ release assay; TST: tuberculin skin test. 


\section{RESULTS}

The targeted sample of 40 consecutive clinical records per study site was collected and, in total, complete data on $200 \mathrm{~TB}$ cases (150 being MDR-TB cases) were available for the analysis. The overall results are summarised in tables 2 and 3, stratified by the main areas surveyed (TB prevention, TB diagnosis and TB treatment). Having previously described the findings within the area of TB prevention in a specifically dedicated publication [25], the following section is focussed on the results within the areas of TB diagnosis and TB treatment.

At the study sites, information on patients' previous medical history was assessed for the majority of cases; investigation of previous TB diagnosis was performed for $93 \%$ (186 out of 200) of the cases, investigation of previous TB treatment for $99 \%$ (198 out of 200) of the cases and investigation of previous drug susceptibility testing (DST) for $94 \%$ (188 out of 200) of the cases surveyed. Microbiological evaluation was missing, or not satisfactory, in $15(7.5 \%)$ out of the 200 clinical files reviewed. The use of correct diagnostic algorithms leading to a bacteriologically confirmed diagnosis was recorded in $94.5 \%$ (189 out of 200) of the evaluated patient records.

$10 \%$ (20 out of 200) of the enrolled individuals were not adequately treated (i.e. the regimen prescribed included fewer than the four recommended drugs). Furthermore, various errors were identified in the doses of anti-TB drugs prescribed (13 out $200,6.5 \%$ ) and, mostly, in the duration of the anti-TB therapy (34 out of $200,16.9 \%$ ).

TB/HIV co-infection was not satisfactorily managed in $34.8 \%$ (eight out of 23) of the cases; in particular, antiretroviral therapy was not prescribed in agreement with existing recommendations $[16,17,21]$. No adverse events were notified in the cases that started combined antiretroviral and anti-TB therapy.

Out of the 200 clinical records examined, 196 (98\%) had a treatment outcome recorded; 109 (55.6\%) out of 196 had a definitive outcome (cure, treatment completed, death or failure). The majority of the remaining cases (63 out of $196,32.1 \%$ ) were commonly defined as being "transferred out", indicating that the clinical reference centres were not aware of the final outcome of the majority of their managed patients, while $16(8.2 \%)$ out of 196 defaulted and six (3.1\%) out of 196 were still on treatment at the time of the survey. Table 2 summarises the results by main variable collected, stratifying data by setting/country, comparing the results between settings/countries and providing overall results. Table 3 presents the specific diagnostic and treatment variables assessed in the survey and the results, for each reference centre included in the study.

In summary, the main areas where disagreement between current established guideline requirements and practices was observed were as follows. Surveillance: lack of information available in the treating clinical reference centre on the final outcome of the discharged patients; clinical management of TB and MDR-TB: incomplete information on previous treatment, inadequate contact tracing procedures, inadequate bacteriological diagnosis, prescription of fewer than four active drugs, incorrect drug choice, inadequate dose prescription, short treatment duration and inadequate management of adverse events of treatment; clinical management of HIV: suboptimal HIV testing and counselling of TB cases, and inadequate prescription of antiretroviral drugs as well as management of combined TB/HIV treatment; laboratory support: suboptimal

TABLE 2 Description of the main findings related to tuberculosis (TB) diagnosis and treatment

\begin{tabular}{|c|c|c|c|c|c|c|}
\hline & $\begin{array}{c}\text { European } \\
\text { cohort }\end{array}$ & $\begin{array}{l}\text { Group } 1 \\
\text { country }\end{array}$ & $\begin{array}{c}\text { Group 2a } \\
\text { country }\end{array}$ & $\begin{array}{c}\text { Group 2b } \\
\text { country }\end{array}$ & $\begin{array}{l}\text { Group } 3 \\
\text { country }\end{array}$ & $\begin{array}{c}\text { Group } 4 \\
\text { country }\end{array}$ \\
\hline \multicolumn{7}{|l|}{ TB diagnosis } \\
\hline Investigation of previous TB diagnosis performed & 186/200 (93) & $40(100.0)$ & $40(100.0)$ & $40(100.0)$ & $40(100.0 .0)$ & $26(65.0)$ \\
\hline Investigation of previous treatment performed & 198/200 (99) & $40(100.0)$ & $40(100.0)$ & $40(100.0)$ & $40(100.0)$ & $38(95.0)$ \\
\hline Investigation of previous DST performed & $188 / 200(94)$ & $40(100.0)$ & $40(100.0)$ & $40(100.0)$ & $40(100.0)$ & $28(70.0)$ \\
\hline Diagnostic algorithm correct & $189 / 200(94.5)$ & $39(97.5)$ & $40(100.0)$ & $40(100.0)$ & $38(95.0)$ & $32(80.0)$ \\
\hline Microbiological analysis performed & $185 / 200(92.5)$ & $40(100.0)$ & $40(100.0)$ & $39(97.5)$ & $30(75.0)$ & $36(90.0)$ \\
\hline Other necessary examinations performed & 196/200 (98) & $40(100.0)$ & $40(100.0)$ & $40(100.0)$ & $36(90.0)$ & $40(100.0)$ \\
\hline Final diagnostic decision taken correctly & $196 / 200(98)$ & $40(100.0)$ & $39(97.5)$ & $39(97.5)$ & $40(100.0)$ & $38(95.0)$ \\
\hline \multicolumn{7}{|l|}{ TB treatment } \\
\hline Correct TB regimen choice ${ }^{\#}$ & $180 / 200(90)$ & $36(90.0)$ & $28(70.0)$ & $38(95.0)$ & $40(100.0)$ & $38(95.0)$ \\
\hline Dosage correct & $187 / 200(93.5)$ & $29(72.5)$ & $40(100.0)$ & $39(97.5)$ & $39(97.5)$ & $40(100.0)$ \\
\hline Duration correct & $166 / 200(83.1)$ & $30(75.0)$ & $18(45.0)$ & $39(97.5)$ & $40(100.0)$ & $39(97.5)$ \\
\hline Management of adverse events due to TB treatment correct & $199 / 200(99.5)$ & $40(100.0)$ & $40(100.0)$ & $40(100.0)$ & $40(100.0 .0)$ & $39(97.5)$ \\
\hline HIV antiretroviral regimen choice correct & $15 / 23(65.2)$ & $2(100.0)$ & & $1(100.0)$ & $12(63.2)$ & $0(0.0)$ \\
\hline Management of adverse events due to HIV treatment correct & $0 / 0(0)$ & $0 / 0(0)$ & $0 / 0(0)$ & $0 / 0(0)$ & $0 / 0(0)$ & $0 / 0(0)$ \\
\hline Correct TB treatment outcome assigned & $196 / 200(98)$ & $40(100.0)$ & $40(100.0)$ & $40(100.0)$ & $37(92.5)$ & $39(97.5)$ \\
\hline
\end{tabular}

Data are presented as n/N (\%) or $n$ (\%). Group 1: former Soviet Union; intermediate TB incidence, high multidrug-resistant (MDR-)TB prevalence. Group 2a: northern Europe; low TB incidence, low MDR-TB prevalence. Group 2b: southern Europe; low TB incidence, low MDR-TB prevalence. Group 3: southern Europe; intermediate TB incidence, low MDR-TB prevalence. Group 4: central Europe; intermediate TB incidence, low MDR-TB prevalence. DST: drug susceptibility testing. \# : four active drugs ensured. 
TABLE 3 Diagnostic and treatment findings evaluated in five national tuberculosis (TB) reference centres

\begin{tabular}{|c|c|c|c|c|c|}
\hline & $\begin{array}{c}\text { Group } 1 \\
\text { country }\end{array}$ & $\begin{array}{c}\text { Group 2a } \\
\text { country }\end{array}$ & $\begin{array}{c}\text { Group 2b } \\
\text { country }\end{array}$ & $\begin{array}{c}\text { Group } 3 \\
\text { country }\end{array}$ & $\begin{array}{c}\text { Group } 4 \\
\text { country }\end{array}$ \\
\hline \multicolumn{6}{|l|}{ Case definition } \\
\hline Susceptible & $10(25.0)$ & $5(12.5)$ & $5(12.5)$ & $8(20.0)$ & $10(25.0)$ \\
\hline MDR & $26(65.0)$ & $22(55.0)$ & $25(62.5)$ & $9(22.5)$ & $28(70.0)$ \\
\hline Previous diagnosis of TB recorded & $9(22.5)$ & $21(56.8)$ & $13(32.5)$ & $22(55.0)$ & $15(37.5)$ \\
\hline Extra-pulmonary TB & $4(10.0)$ & $1(2.5)$ & $5(12.5)$ & $0(0.0)$ & $2(5.0)$ \\
\hline Positive microscopy result & $19(47.5)$ & $23(57.5)$ & $32(80.0)$ & $39(97.5)$ & $30(75)$ \\
\hline NAAT performed within the diagnostic algorithm for active TB & $31(77.5)$ & $7(18.0)$ & $39(97.5)$ & $14(35)$ & $0(0)$ \\
\hline TST performed within the diagnostic algorithm for active TB & $15(37.5)$ & $1(2.5)$ & $3(7.9)$ & $0(0.0)$ & $0(0.0)$ \\
\hline Positive IGRA & & & $38(97.4)$ & & \\
\hline Previous TST results recorded & $0(0.0)$ & $0(0.0)$ & $1(2.5)$ & $0(0.0)$ & $0(0.0)$ \\
\hline Previous IGRA results recorded & $0(0.0)$ & $0(0.0)$ & $1(2.5)$ & $0(0.0)$ & $0(0.0)$ \\
\hline Strains resistant to fluoroquinolones & $4(13.3)$ & $4(12.5)$ & $5(18.5)$ & $23(74.2)$ & $2(6.9)$ \\
\hline Strains resistant to second-line injectable drugs & $14(46.7)$ & $6(20.0)$ & $12(44.4)$ & $24(72.4)$ & $13(44.8)$ \\
\hline Number of previous anti-TB treatment regimens $>1$ month & $1.2 \pm 0.44$ & $2 \pm 0.87$ & $1.4 \pm 0.67$ & $1.5 \pm 0.96$ & $2.1 \pm 1.2$ \\
\hline HIV test offered & $39(97.5)$ & $40(100.0)$ & $37(92.5)$ & $23(57.5)$ & $19(47.5)$ \\
\hline HIV-positive cases at current diagnosis & $2(5.1)$ & $0(0.0)$ & $1(2.5)$ & $19(48.7)$ & $1(7.1)$ \\
\hline Males & $23(57.5)$ & $40(100.0)$ & $24(60.0)$ & $32(80.0)$ & $22(55.0)$ \\
\hline Age of patients surveyed in the reference centre yrs & $45.4 \pm 14.8$ & $37.7 \pm 12.4$ & $37.3 \pm 15.5$ & $42.2 \pm 11.8$ & $33.9 \pm 14.0$ \\
\hline Antiretroviral treatment & $2(100.0)$ & & $1(100.0)$ & $12(63.2)$ & $0(0.0)$ \\
\hline Dosages of antiretroviral drugs adequate & $2(100.0)$ & & $1(100.0)$ & $12(63.2)$ & $0(0.0)$ \\
\hline Patients who experienced adverse events of antiretroviral drugs & $0(0.0)$ & & $0(0.0)$ & $0(0.0)$ & $0(0.0)$ \\
\hline Surgery performed for current diagnosis & $4(10.0)$ & $1(2.5)$ & $2(4.1)$ & $2(5.0)$ & $1(2.5)$ \\
\hline \multicolumn{6}{|l|}{ Laserson's criteria" for treatment outcome } \\
\hline Cured & $19(63.3)$ & $3(11.5)$ & $2(8.0)$ & $9(34.6)$ & $20(69.0)$ \\
\hline Completed & $1(3.3)$ & & & $5(19.2)$ & \\
\hline Died & $4(13.3)$ & $1(3.9)$ & $2(8.0)$ & $6(23.1)$ & $3(10.4)$ \\
\hline Defaulted & $6(20.0)$ & $1(3.9)$ & & $5(19.2)$ & $4(13.8)$ \\
\hline Transferred out & & $21(80.8)$ & $20(80.0)$ & $1(3.9)$ & $2(6.9)$ \\
\hline Failed & & & $1(4.0)$ & & \\
\hline Time to SS conversion days from start treatment & $45(34-68)$ & $70(30-150)$ & $49(30-100)$ & $108(65-286)$ & $32(30-60)$ \\
\hline Time to culture conversion days & $58(38-87)$ & $60(40-120)$ & $81(49-130)$ & $94(65-286)$ & $60(30-78)$ \\
\hline
\end{tabular}

Data are presented as $n(\%)$, median (interquartile range) or mean \pm SD. Group 1: former Soviet Union; intermediate TB incidence, high multidrug-resistant (MDR-)TB prevalence. Group 2a: northern Europe; low TB incidence, low MDR-TB prevalence. Group 2b: southern Europe; low TB incidence, low MDR-TB prevalence. Group 3: southern Europe; intermediate TB incidence, low MDR-TB prevalence. Group 4: central Europe; intermediate TB incidence, low MDR-TB prevalence. XDR: extensively drug-resistant; NAAT: nucleic acid amplification test; TST: tuberculin skin test; IGRA: interferon- $\gamma$ release assay; SS: sputum smear. \#: QuantiFERON ${ }_{\mathbb{B}-T B}$ Gold is manufactured by Cellestis (Chadstone, Australia). ${ }^{\prime}$ : outcomes for MDR-TB cases derived from [23]; note that in some low-incidence countries, as many TB deaths occur in the elderly, deaths are divided into death due to TB and death from other causes. 
laboratory practices, particularly for second-line drugs, and lack of DST procedures for some of the second-line drugs; diagnostic and treatment algorithms: relevant inter- and intracountry differences in the diagnostic use of the Mantoux test and new diagnostics, including nucleic acid amplification tests and interferon- $\gamma$ release assays, and inadequate selection of drugs to be tested by DST, as well as choice of second-line drugs used to treat MDR- and XDR-TB cases.

\section{DISCUSSION}

The aim of the study was to investigate the management of TB and MDR-TB in a sample of MDR-TB reference centres in a selection of EU/EEA countries. The results of the study identified areas of priority for public health action.

With regard to ensuring the prevention of nosocomial spread of drug-resistant Mycobacterium tuberculosis strains, the main area of concern was the observed lack of a comprehensive infection control strategy or plan in all the reference centres surveyed [22]. Furthermore, administrative measures, such as infection control committees, were not systematically in place.

The final outcome of discharged patients should be available in the treating clinical centre, as this represents a key managerial and/or monitoring tool; these data were lacking, to a certain extent, in all surveyed centres. Availability of quality outcomes motivates physicians, stimulates critical review of the diagnostic and treatment procedures implemented, and allows continuous quality improvement within the programme [17, 18].

From the diagnostic point of view, several findings deserve comment. The variables assessed in this study reflect data that are routinely reported to, and for, official European surveillance databases and reports: reports in which bacteriological confirmation of TB cases is shown to remain suboptimal in the EU and its member states [3]. A surprising finding was the irregular use of the rapid molecular diagnostic methods [26, 27]. In a setting such as the EU, with greater resources, especially at the reference centre level, it was noteworthy that these assays, which allow for the rapid identification of $M$. tuberculosis and a proxy of MDR-TB (in $<1$ day), are not routinely used in some of the reference centres. This suggests that treatment management is being blindly initiated, in a number of cases, at the sites, and that several patients are isolated for longer periods while waiting for DST results from liquid, and sometimes solid, media culture. The gold standard for diagnosing drug-resistant TB remains that of culture-based DST. However, the rapid molecular methods offer the potential to give a first, essential indication of $M$. tuberculosis and drug-resistance pattern in a patient, enabling rapid, more optimised treatment choices, as well as appropriate infection control measures for each patient. It is important to note that the new diagnostic tools should only be promoted based on clear, evidence-based guidelines [17, 18].

With regard to treatment practices, the choice of treatment regimens, the doses of administered anti-TB drugs and the duration of anti-TB drug treatment did not follow international recommendations in a number of the reference centres. Furthermore, in a few cases, mistakes in managing adverse events, which could lead to the development of further drug resistance, were observed. These findings show that clinical TB and MDR-TB management guidance needs to be strengthened and urgently implemented in the EU setting. This is essential to ensure that complete data on previous treatment(s), adequate contact tracing procedures, adequate bacteriological diagnosis, prescription of at least four active drugs, correct drug choice, adequate dose prescription, sufficient treatment duration and adequate management of treating adverse events are secured for every TB patient [21].

Our findings further present the need to strengthen the clinical management of HIV and the need to develop TB/HIV collaborative activities in Europe [28-31]. This is particularly relevant in the areas of HIV testing and counselling of TB cases, antiretroviral prescription and combined TB/HIV treatment management $[17,18]$.

The current study and its findings emphasise the importance of devoting financial support and human resources to ensure optimal clinical training and retraining of all staff categories involved in TB control, particularly in the present situation of a progressive decline in TB incidence and, thus, decrease in clinical expertise in some parts of Europe. To ensure optimal MDR-TB case management, prevention and control, this study confirms the importance and need to define clear policies supporting the admission of MDR-TB and "difficult" TB cases to specialised reference institutions in Europe. Lastly, the survey identified a clear gradient, in terms of number and severity of drawbacks between peripheral centres, in and outside EU/EEA countries (where most of the case management mistakes occurred and were subsequently identified when analysing the original records in the reference centres), and the reference centres surveyed in this study [14].

\section{Conclusions}

This survey identified relevant mistakes and deviations from the recommended practices in the management of TB and MDR/XDR-TB cases in Europe, even at the level of national clinical reference centres. Each individual error in managing an MDR-TB case can be seen as a potential for generating a new MDR-TB case and highlights the relevance of each of the errors. Furthermore, considering that the pitfalls described were identified in national reference centres in Europe, their relevance appears more alarming as it raises concern with regard to TB case management at the peripheral level. Indeed, the situation in peripheral health services was reported to be worse in all the countries surveyed (data not shown) [14].

The challenges identified in this survey can be divided into two separate parts: 1) challenges related to the clinical mismanagement of individual cases, leading potentially to the further selection of resistant strains; and 2) public health challenges, related to policy, legal, financial and organisational aspects of TB and MDR-TB prevention and control. Between these two parts, there were structural weaknesses of the health units hosting the surveyed national reference centres: for example, the lack of negative-pressure ventilation rooms or the lack of respiratory isolation rooms.

In conclusion, the results of this survey identify the following areas to be strongly considered and discussed among partners and stakeholders. 1) Surveillance: data on patients' final outcome need to be available at the clinical centre as a managerial and quality improvement tool; 2) infection control: implementation of national and facility plans, which include 
the need for respiratory isolation, negative-pressure ventilation rooms (if really necessary), standardised health education, masks for patients and respirators for staff, respirator fit testing, and appropriate training; 3) clinical management of TB: accurate and complete knowledge of previous treatment, adequate contact tracing procedures [31, 32], adequate bacteriological diagnosis (including use of rapid molecular tests), prescription of at least four active drugs, correct drug choice, correct dose prescription, adequate treatment duration, and adequate management of adverse events; 4) clinical management of HIV: HIV testing and counselling, antiretroviral prescription, and combined TB/HIV treatment management; 5) laboratory support: optimal laboratory practices and quality assurance procedures, particularly for second-line drugs at the reference centre level and implementation of methods described under areas 2, 3 and 6; 6) diagnostic and treatment algorithms: increased implementation of new diagnostic tools, e.g. rapid molecular DST, within clear evidence-based guidelines; 7) guidelines: adoption of specific, updated, evidence-based TB and MDR-TB guidelines in all European countries and development of European Standards for TB and MDR-TB prevention and control; 8) drugs: availability of all first- and second-line drugs in all European countries; 9) funding: consistent funding resources for TB and MDR-TB prevention and control in all European countries.

A response to this need has already been initiated through the development of European Union Standards for TB Care. Identifying key standards for the diagnosis, management, prevention and control of TB, MDR-TB and XDR-TB, these standards aim at supporting healthcare workers in optimising TB case management and, thus, at contributing to improved TB control in the EU/EEA [33].

It should be recognised that only through the achievement of excellence and optimal care for $\mathrm{TB}$, particularly its most resistant forms, can we hope to start curbing the epidemic towards its elimination [34].

\section{SUPPORT STATEMENT}

The study reported in this manuscript was financially supported through European Centre for Disease Prevention and Control Direct Service Contract (publication reference OJ/2009/06/24-PROC/2009/ 030), awarded to the Tuberculosis Network European Trials Group (Bad Oldesloe, Germany).

\section{STATEMENT OF INTEREST}

None declared.

\section{REFERENCES}

1 World Health Organization. Multidrug and extensively drugresistant TB (M/XD-TB). 2010 Global report on surveillance and response. Document WHO/HTM/TB/2010.3. Geneva, World Health Organization, 2010.

2 World Health Organization. Anti-tuberculosis drug resistance in the world. Fourth global report. Document WHO/HTM/TB/ 2008.394. Geneva, World Health Organization, 2008.

3 European Centre for Disease Prevention and Control, World Health Organization Regional Office for Europe. Tuberculosis Surveillance in Europe 2009. Stockholm, European Centre for Disease Prevention and Control, 2011.

4 Sotgiu G, Ferrara G, Matteelli A, et al. Epidemiology and clinical management of XDR-TB: a systematic review by TBNET. Eur Respir J 2009; 33: 871-881.
5 Migliori GB, Loddenkemper R, Blasi F, et al. 125 years after Robert Koch's discovery of the tubercle bacillus - the new XDR-TB threat. Is "science" enough to tackle the epidemic? Eur Respir J 2007; 29: 423-427.

6 Migliori GB, Besozzi G, Girardi E, et al. Clinical and operational value of the extensively drug-resistant tuberculosis definition. Eur Respir J 2007; 30: 623-626.

7 Migliori GB, Ortmann J, Girardi E, et al. Extensively drug-resistant tuberculosis, Italy and Germany. Emerg Infect Dis 2007; 13: 780-782.

8 Migliori GB, Lange C, Centis R, et al. Resistance to second-line injectables and treatment outcomes in multidrug-resistant and extensively drug-resistant tuberculosis cases. Eur Respir J 2008; 31: 1155-1159.

9 Migliori GB, Lange C, Girardi E, et al. Fluoroquinolones: are they essential to treat multidrug-resistant tuberculosis? Eur Respir J 2008; 31: 904-905.

10 Migliori GB, Richardson MD, Lange C. Of blind men and elephants: making sense of extensively drug-resistant tuberculosis. Am J Respir Crit Care Med 2008; 178: 1000-1001.

11 World Health Organization. Extensively drug-resistant tuberculosis (XDR-TB): recommendations for prevention and control. Wkly Epidemiol Rec 2006; 81: 430-432.

12 Migliori GB, Sotgiu G, Jaramillo E, et al. Development of a standardised multidrug-resistant/extensively drug-resistant tuberculosis assessment and monitoring tool. Int J Tuberc Lung Dis 2009; 13: 1305-1308.

13 Migliori GB, Hopewell PC, Blasi F, et al. Improving the TB case management: the International Standards for Tuberculosis Care. Eur Respir J 2006; 28: 687-690.

14 Sotgiu G, Centis R, D'Ambrosio L, et al. Development of a standardised tool to survey MDR-/XDR-TB case management in Europe. Eur Respir J 2010; 36: 208-211.

15 National Collaborating Centre for Chronic Conditions. Tuberculosis: clinical diagnosis and management of tuberculosis, and measures for its prevention and control. London, Royal College of Physicians, 2006.

16 Tuberculosis Coalition for Technical Assistance. International Standards for Tuberculosis Care (ISTC). The Hague, Tuberculosis Coalition for Technical Assistance, 2006.

17 Tuberculosis Coalition for Technical Assistance. International Standards for Tuberculosis Care (ISTC), 2nd Edn. The Hague, Tuberculosis Coalition for Technical Assistance, 2009.

18 WHO. Treatment of Tuberculosis: Guidelines for National Programmes. Document WHO/CDS/TB/2003.313. Geneva, World Health Organization, 2003.

19 World Health Organization. Treatment of tuberculosis guidelines. 4th Edn. Document WHO/HTM/TB/2009.420. Geneva, World Health Organization, 2010.

20 USAID-PATH MDR/XDR-TB Assessment and Monitoring Tool. www.path.org/publications/details.php? $i=1678$ Date last accessed 2009. Date last updated: September 2011.

21 World Health Organization. Guidelines for the programmatic management of drug-resistant tuberculosis. Document WHO/ HTM/TB/2008.402. Geneva, World Health Organization, 2008.

22 World Health Organization. WHO policy on infection control in health-care facilities, congregate settings and households. Document WHO/HTM/TB/2009.419. Geneva, Health Organization, 2009.

23 Laserson $\mathrm{K}$, Thorpe LE, Leimane V, et al. Speaking the same language: treatment outcome definitions for multidrug-resistant tuberculosis. Int J Tuberc Lung Dis 2005; 9: 640-645.

24 Broekmans JF, Migliori GB, Rieder HL, et al. European framework for tuberculosis control and elimination in countries with a low incidence. Recommendations of the World Health Organization (WHO), International Union against Tuberculosis and Lung Disease (IUATLD) and Royal Netherlands Tuberculosis Association (KNCV) Working Group. Eur Respir J 2002; 19: 765-775. 
25 Sotgiu G, D'Ambrosio L, Centis R, et al. TB and M/XDR-TB infection control in European TB reference centres: the Achilles' heel? Eur Respir J 2011; 38: 1221-1223.

26 World Health Organization. Report of the Tenth Meeting WHO Strategic and Technical Advisory Group for Tuberculosis (STAGTB) 27-29 September 2010. Document WHO/HTM/TB/2010.18 Geneva, World Health Organization, 2010.

27 Boehme CC, Nicol MP, Nabeta P, et al. Feasibility, diagnostic accuracy, and effectiveness of decentralised use of the Xpert MTB/RIF test for diagnosis of tuberculosis and multidrug resistance: a multicentre implementation study. Lancet 2011; 377: 1495-1505.

28 Sester M, Giehl C, McNerney R, et al. Challenges and perspectives for improved management of HIV/Mycobacterium tuberculosis coinfection. Eur Respir J 2010; 36: 1242-1247.

29 Kruijshaar ME, Pimpin L, Abubakar I, et al. The burden of TBHIV in the EU: how much do we know? A survey of surveillance practices and results. Eur Respir J 2011; 38: 1374-1381.

30 Pimpin L, Drumright LN, Kruijshaar ME, et al. Tuberculosis and HIV co-infection in European Union and European Economic Area countries. Eur Respir J 2011; 38: 1382-1392.

31 Pontali E, Pasticci MB, Matteelli A, et al. Tuberculosis and HIV coinfection: do we have a surveillance system in Europe? Eur Respir J 2011; 38: 1258-1260.

32 Erkens CG, Kamphorst M, Abubakar I, et al. Tuberculosis contact investigation in low prevalence countries: a European consensus. Eur Respir J 2010; 36: 925-949.

33 Migliori GB, Sotgiu G, Blasi F, et al. Towards the development of EU/EEA Standards for Tuberculosis Care (ESTC). Eur Respir J 2011; 38: 493-495.

34 Falzon D, Jaramillo E, Schünemann HJ, et al. WHO guidelines for the programmatic management of drug-resistant tuberculosis: 2011 update. Eur Respir J 2011; 38: 516-528. 